\title{
¡ㄱㅌㄷI\&NE
}

\section{Análisis de tendencias en la recuperación de suelos empleando vigilancia tecnológica}

\author{
Analysis of trends in soil recovery using technological surveillance

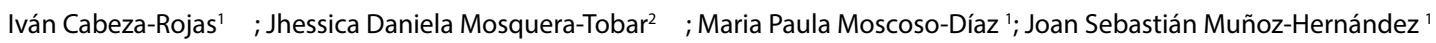 \\ 1 Politécnico Grancolombiano, Bogotá, Colombia. icabeza@poligran.edu.co; mamoscoso2@poligran.edu.co; jomunozh@poligran.edu.co \\ 2 Universidad Nacional de Colombia, Bogotá, Colombia. jhmosquerat@unal.edu.co
}

Fecha de recepción: 21 de septiembre 2021. Fecha de aprobación: 19 de noviembre de 2021

\begin{abstract}
Resumen- Las actividades antropogénicas han afectado recursos naturales limitados, como el recurso suelo, el cual soporta diferentes actividades que se pueden considerar indispensables para la humanidad. En este sentido, la salud del suelo es ampliamente estudiada desde lo académico, especialmente en el contexto de la recuperación de suelos degradados. Así, el presente documento desarrollo una vigilancia tecnológica que permitirá analizar la información disponible relacionada a técnicas de recuperación de suelos degradados, lo que permite presentar un contexto general, las técnicas y estudios más significativos del área. La vigilancia se realizó empleando las bases de datos de Scopus y ESPACENET, que corresponden a la búsqueda de artículos científicos y patentes, respectivamente. Se evaluaron indicadores relacionados al número de publicaciones, tipo de publicación, registro de patentes por país, entre otros. Se encontraron más de 22.907 artículos y 380 patentes que correspondían al objeto de la búsqueda. Estas investigaciones e invenciones se relacionan, en su mayoría, a procesos de fitorremediación enfocados en la problemática de contaminación de suelos por metales pesados, y se desarrollan en países como China y Estados Unidos, lo que se asocia al crecimiento de procesos agroindustriales altamente tecnificados, la mitigación de la degradación por contaminación antrópica y la búsqueda del desarrollo sostenible.
\end{abstract}

Palabras clave- recuperación de suelos, enmiendas, manejo de la información, vigilancia tecnológica, bibliométrica.

Abstract- Anthropogenic activities have affected limited natural resources, such as the soil, which supports different activities considered essential for humanity. Consequently, soil health is widely studied by academia, especially in the recovery of degraded soils. Thus, this document developed a technological surveillance that will allow the analysis of the available information related to recovery of degraded soils techniques; this will allow to present a general context, the relevant techniques, and studies in the area. Technological surveillance was carried out using Scopus and ESPACENET databases, which regard to the search for scientific articles and patents, respectively. Different bibliometric indicators were evaluated, such as: number of publications, type of publication, patent registration per country, among others. More than $\mathbf{2 2 . 8 0 0}$ articles and 380 patents were found that matched the object of the search. These investigations and inventions are mostly related to phytoremediation processes focused on soil contamination by heavy metals issues and developed in China and the United States, this is related to the growth of intensive technification of agroindustry, the mitigation of the degradation of the soil resource by anthropic contamination, and the sustainable development objectives.

Keywords- soil recovery, organic amendment, information management, technological surveillance, bibliometrics.

\section{INTRODUCCIÓN}

La contaminación ambiental alcanza los recursos aire, agua y suelo en diferentes grados y con diferentes efectos en el desarrollo de actividades antropogénicas actuales. El impacto ambiental a estos recursos nace de su aprovechamiento, el uso de los bienes

Citar este artículo como: I. Cabeza Rojas, J. Mosquera Tobar, M. Moscoso Díaz, y J. Muñoz Hernández, Análisis de tendencias en la recuperación de suelos empleando vigilancia tecnológica, ITECKNE, 19(1), 2022. pp. 39- 45 Doi: https://doi.org/10.15332/iteckne.v19i1.2634 
y servicios generados, y la disposición de residuos [1]. Estos recursos presentan múltiples contaminantes, tantos como las actividades que los aprovechan. Empero, desde el concepto del desarrollo sostenible se hace importante generar alternativas que reduzcan y mitiguen los impactos negativos derivados de las actividades antropogénicas; esto puede ser abordado desde un punto de vista holístico o recurso por recurso, por lo que desde la industria y la academia se presentan alternativas que van desde cambios en los procesos productivos hasta procesos de remediación. Por su parte, el suelo es considerado un punto crítico para lograr los objetivos de desarrollo sostenible -ODS-, por lo que se incentiva el desarrollo científico entorno a este recurso [2].

El suelo, como recurso natural esencial, es un sistema complejo de materia orgánica e inorgánica donde se desarrolla la vida [3], [4]. La mayoría de la investigación académica acerca del recurso suelo está asociada a su importancia en la cadena alimenticia, sin suelos sanos, no serían posibles los sistemas de producción agroindustrial. Adicionalmente, de la calidad de este recurso depende el desarrollo de diferentes ecosistemas; incluyendo la concepción del suelo como asentamiento de la sociedad humana. Así, como recurso natural esencial, el suelo es sujeto de procesos de manejo y mitigación asociados al ordenamiento territorial, la recepción de desechos, y para el manejo del cambio climático [5].

Entre los aspectos negativos que se estudian en torno al recurso suelo se destacan: a) acidificación, que limitan la actividad de micro-y meso-organismos; b) contaminación por acumulación de metales pesados ( $\mathrm{Cd}, \mathrm{Cr}, \mathrm{Cu}, \mathrm{Pb}, \mathrm{Hg}, \mathrm{Ni}, \mathrm{Zn}, \mathrm{Tl}$, y As), su consumo es peligroso y son objeto de bioacumulación a través de la cadena trófica y el agua; c) salinización, la acumulación de sales de sodio afecta el crecimiento de las plantas; c) erosión, afecta la fertilidad y capacidad de retención de agua [3]. Asimismo, entre las actividades que producen impactos negativos se destacan: actividades antropogénicas derivadas de procesos de industrialización, industrialización agrícola, disposición de residuos y urbanización [6].

Fig.1. TIPOS, CAUSAS Y CLASIFICACIONES ASOCIADAS A LA DEGRADACIÓN DEL SUELO.

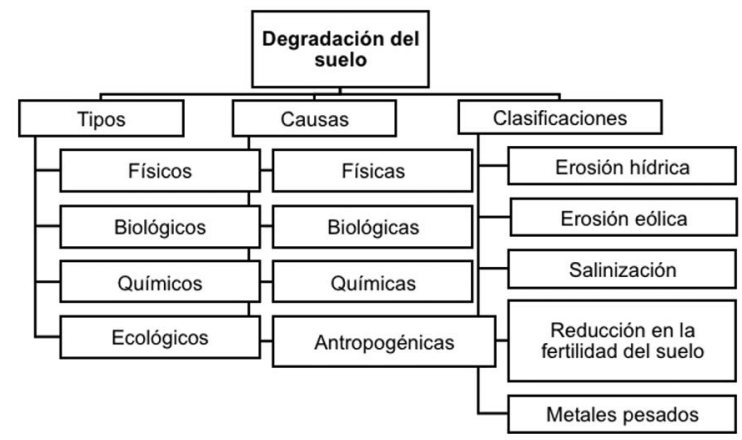

Fuente: Adaptado [7].

Por otro lado, los procesos de remediación estarán asociados a tipos, causas y clasificaciones de los procesos de degradación del suelo, Fig.1. Las estrategias de recuperación de suelo responden principalmente a la clasificación de degradación: a) para suelos erosionados existen estrategias de estabilización y control temporal de sedimento. b) para suelos salinizados se presentan estrategias de drenado e irrigación, y adición de enmiendas orgánicas e inorgánicas. c) para el incremento de la fertilidad del suelo, se proponen balances en el uso de fertilizantes, la estrategia 4Rs (por su origen anglo: right source, right amount, right application time, and right place), además se recomienda la no labranza de los suelos afectados. Por último, d) para la restauración de suelos afectados por metales pesados se plantean estrategias de manejo agrícola enfocadas en procesos de irrigación, fertilización y sistemas de cultivos intercalados; asimismo, la aplicación acondicionadores orgánicos (uso de compostaje); además, enmiendas orgánicas e inorgánicas, biorremediación y fitorremediación.

\section{Vigilancia tecnológica}

Al abordar la investigación en torno a los procesos de contaminación y recuperación del recurso suelo, se requiere de la utilización de metodologías como la vigilancia tecnológica que permitan gestionar el alto número de información relacionada a este recurso. La vigilancia tecnología es consecuencia del surgimiento de sistemas de información, el internet y la invención de patentes como se expone en el Acuerdo sobre los Aspectos de los Derechos de Propiedad Intelectual relacionados con el Comercio - ADPIC, constituidos en 1994, surgieron los elementos que definen la vigilancia tecnológica como una práctica que facilita a los no expertos el tratamiento de la información. Esta metodología permite realizar una búsqueda sistemática, orientada al análisis de información científica y tecnológica específica, que permite sintetizar la información que se tiene actualmente sobre el tema de interés [8].

Ahora bien, la información relacionada a procesos de contaminación y recuperación del recurso suelo data de 1976, donde se encuentra información sobre el manejo de residuos nucleares, y no es sino hasta mediados del 2000 que comienza a hacer un argumento más relevante. Por esto, durante casi 3 décadas las investigaciones, en su mayoría, se dedicaron a encontrar nuevas técnicas de mitigación y recuperación enfocadas en la parte práctica; así, después de tener el interés científico y gubernamental se lograron avances tanto en la publicación académica como en la creación de patentes. Por lo que, se espera un alto número de publicaciones relacionadas.

De acuerdo con lo anterior, el presente documento tuvo como objeto presentar un análisis bibliométrico y patentométrico de las técnicas más estudiadas y aplicadas en recuperación de suelos degradados por causas antrópicas. Este análisis se llevó a cabo reconociendo las palabras clave asociadas al tema de estudio, realizado una búsqueda en las bases de datos de Scopus y ESPACENET, y evaluando la información resultante.

\section{METODOLOGÍA}

El desarrollo de la perspectiva teórica en la investigación científica debe darse en dos etapas: revisión analítica de la 
literatura correspondiente y la construcción de un marco teórico [9]. En este sentido, la revisión analítica corresponde a la una vigilancia tecnológica, que es a su vez una revisión selectiva donde se extrae y recopila información bibliográfica relevante para el desarrollo de la investigación. Por otra parte, la norma UNE - Gestión de la I+D+i: Sistema de vigilancia e inteligencia se centra en la captura de información, la selección y el análisis, la difusión y comunicación de conocimiento para la toma de decisiones en una organización, la metodología establecida, Fig. 2.

Fig. 2. CICLO DE VIGILANCIA PLANTEADO POR LA NORMA UNE

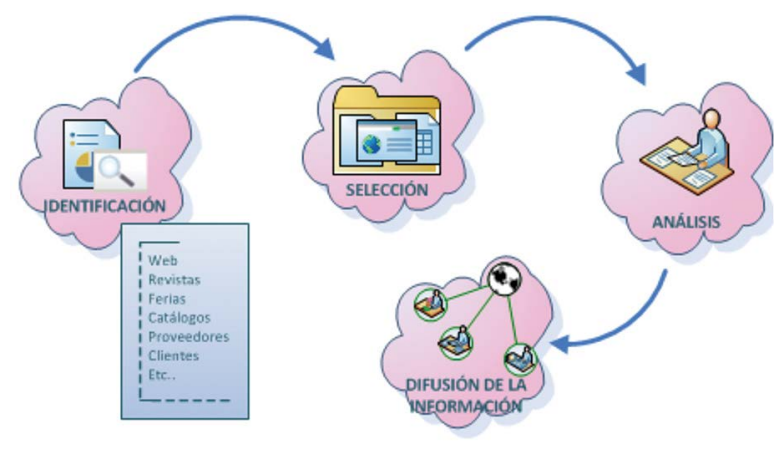

Fuente: Tomado de [8].

Así, de acuerdo con lo establecido la vigilancia tecnológica a desarrollar comprendió tres fases fundamentales:

a) planeación e identificación de necesidades: consistió en la identificación de un objetivo, determinar las fuentes de información a consulta, y establecer una estrategia de búsqueda.

b) identificación, búsqueda y captación de información: incluyo establecer las palabras clave asociadas al proyecto, a través de una búsqueda parcial en plataformas como Google Académico. Lo que permitió construir una ecuación de búsqueda que se empleó en bases de Scopus y ESPACENET. Por último, se realizó una verificación de la relevancia de los datos.

c) organización, depuración y análisis de la información: se construyeron indicadores bibliométricos y cienciométricos para la gestión de la información desde un análisis de tendencias.

La vigilancia tecnológica tuvo el objeto de analizar las técnicas de recuperación del recurso suelo más estudiadas en los últimos años. En este sentido, se recurrió a la búsqueda de información en buscadores como Google Académico para establecer las palabras clave que respondieran a los aspectos más relevantes encontrados en la literatura académica en torno al tema de estudio; conjuntamente, se buscaba que dichas palabras clave fueran recurrentes.

Posteriormente, se plantearon ecuaciones de búsqueda que permitieran la identificación de los avances en cada técnica seleccionada y los principales autores en el campo. Estas ecuaciones se construyeron con operadores booleanos, los cuales conectan las palabras clave haciendo más eficiente la búsqueda al mejorar la integración de los tesauros, entre ellos y con sus sinónimos [10]. Por otro lado, una vez seleccionada la mejor ecuación de búsqueda, basados en el número de publicaciones relevantes y su relación con el objeto de estudio, se realizó un análisis bibliométrico empleando herramientas de Scopus y ESPACENET.

Estas plataformas desarrollan análisis bibliométricos de las ecuaciones de búsqueda ingresadas, lo que permite verificar: autores relevantes, número de citaciones e impacto de las publicaciones, países con mayor número de publicaciones, revistas científicas asociadas al tema de estudio, tendencias de publicación, entre otros aspectos. Asimismo, la plataforma Scopus cuenta con filtros que permiten identificar: los documentos más relevantes, los documentos más citados y los más recientes. Lo cual resulta útil en el proceso de organización, depuración y análisis de la información, pudiendo seleccionar de forma más eficiente los documentos más relevantes según el objeto de estudio.

Cabe resaltar que la búsqueda de patentes se trasladó a la base de datos ESPACENET, donde se realizó una búsqueda de códigos IPC para seleccionar los códigos relacionados con mayor número de publicaciones. Esto permitió generar nuevas ecuaciones de búsqueda, solo asociadas a este análisis patento métrico.

\section{RESULTADOS}

La identificación inicial de palabras clave se llevó a cabo en buscadores como Google Académico, donde se inicia la búsqueda en torno a las técnicas de recuperación del recurso suelo, allí, a nivel general, los tesauros más empleados para referirse a esta temática se relacionan en la Tabla I.

TABLA I.

PALABRAS CLAVE PARA LA CONSTRUCCIÓN DE LA ECUACIÓN DE BÚSQUEDA.

\begin{tabular}{|c|c|c|}
\hline Palabra Clave & Key Word & Synonym \\
\hline Suelo & soils & land \\
\hline Recuperación & recovery & $\begin{array}{c}\text { remediation, } \\
\text { restoration }\end{array}$ \\
\hline Contaminante & contaminant & pollutant \\
\hline Fertilidad & fertility & -- \\
\hline Fertilizante & fertilizer & -- \\
\hline Biorremediación & bioremediation & -- \\
\hline Fitorremediación & phytoremediation & Phytotechnology \\
\hline Compostaje & compost & -- \\
\hline Biomasa & biomass & biochar \\
\hline Enmienda & amendment & - \\
\hline Conservación & conservation & - \\
\hline
\end{tabular}




\subsection{Evaluación referencial con la herramienta de Scopus}

La identificación de técnicas y palabras claves permitieron evaluar diferentes ecuaciones de búsqueda en la herramienta Scopus, Tabla II. Esto se hizo con el fin de realizar un filtro intermedio de 4 posibles ecuaciones de búsqueda; donde la mejor ecuación de búsqueda bibliométrica fue seleccionada verificando el número de documentos respecto a la representatividad de estos en torno al objetivo propuesto. Así, la ecuación de búsqueda seleccionada fue la número 5, donde se encontraron publicaciones relevantes de acuerdo con el objetivo planteado, además de ser una ecuación con alta representatividad en los términos seleccionados, sin tener un tamaño de muestra excesivo. Por otro lado, en Scopus, se encontraron 12.662 patentes para la misma ecuación de búsqueda.

TABLA II.

ECUACIONES DE BÚSQUEDA EVALUADAS EN SCOPUS.

\begin{tabular}{|c|c|}
\hline Ecuación & Resultado \\
\hline $\begin{array}{l}\text { 1. ("soil recovery" AND ("fertilizer" OR "composting" OR } \\
\text { "biomass" OR "conservation")) }\end{array}$ & 146 \\
\hline $\begin{array}{l}\text { 2. (soil AND conservation AND ("fertilizer" OR } \\
\text { "composting" OR "biomass" OR "organic } \\
\text { amendment" OR "biochar" OR "phytotechnology" OR } \\
\text { "bioremediation")) }\end{array}$ & 9.035 \\
\hline $\begin{array}{l}\text { 3. (soil AND recovery AND ("organic matter" OR } \\
\text { "organic pollutant" OR "inorganic pollutant" OR } \\
\text { "decontamination") AND ("microorganisms" OR } \\
\text { "compost")) }\end{array}$ & 427 \\
\hline $\begin{array}{l}\text { 4. (soil AND ("compost" OR "biomass") AND } \\
\text { ("conservation" OR "amendment" OR "biochar") AND } \\
\text { ("phytoremediation" OR "bioremediation")) }\end{array}$ & 1.343 \\
\hline $\begin{array}{l}\text { 5. (soils AND (("pollutant" OR "conservation") AND } \\
\text { ("compost" OR "fertilizer" OR "phytoremediation" } \\
\text { OR "bioremediation" OR "organic amendment" OR } \\
\text { "biochar"))) }\end{array}$ & 24.736 \\
\hline
\end{tabular}

Haciendo uso de las herramientas de análisis de Scopus, se encontró que entre los países con mayor cantidad de publicaciones se encuentran China y Estados Unidos, seguidos por India, España y Reino Unido; siendo estos los que lideran los reportes en más del $80 \%$. Por su parte, Colombia se encuentra en la posición 55 con 68 publicaciones.

Las revistas que tienen mayor número de publicaciones asociadas con la búsqueda son: Chemosphere, Science Of The Total Environment, Environmental Science And Pollution Research, Journal Of Hazardous Materials, y Environmental Pollution. Lo que a su vez implica, un mayor número de citaciones asociadas al tema de estudio, debido al factor de impacto de dichas revistas científicas, 215 en promedio de índice de impacto para las revistas con mayor número de publicaciones de acuerdo con la ecuación de búsqueda se- leccionada. Así mismo, entre los investigadores destacados se encuentran: Ok, Y.S, Naidu, R., Christie, P. y Luo, Y.; quienes cuentan con más de 80 publicaciones relacionadas. Para este caso, las palabras claves que se asocian a estos autores son: fitorremediación, biorremediación, enmiendas biológicas, biochar, solidos contaminados, biomasa; las cuales coinciden con la ecuación de búsqueda seleccionada.

Igualmente, a pesar de que la investigación relevante inicia en los años 1970, se observó que el auge de la recuperación de suelos y la publicación científica en estos temas se da desde 2000, aumentando exponencialmente como respuesta a la creciente interés en la contaminación ambiental por metales pesados y su bioacumulación [11], [12], como se muestra en la Fig. 3.

Fig. 3. HISTOGRAMA DE PUBLICACIONES SEGÚN ECUACIÓN DE BÚSQUEDA.

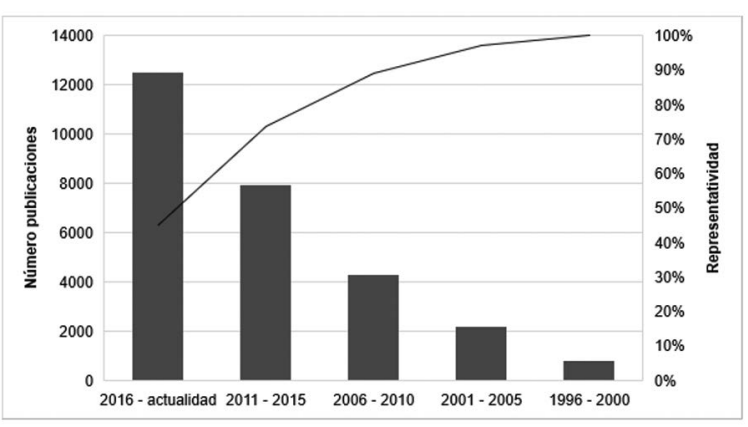

Fuente: Los autores

Cabe resaltar que, a la fecha de divulgación del artículo, la tipología de los documentos encontrados se divide de la siguiente forma: $80 \%$ artículos de investigación, $7.4 \%$ artículos de conferencias, $6.7 \%$ artículos de revisión, $3.6 \%$ capítulos de libro, $1.5 \%$ entre libros, notas y otros documentos, Fig. 4.

Fig. 4. DISTRIBUCIÓN PORCENTUAL DE DOCUMENTOS PUBLICADOS POR TIPO.
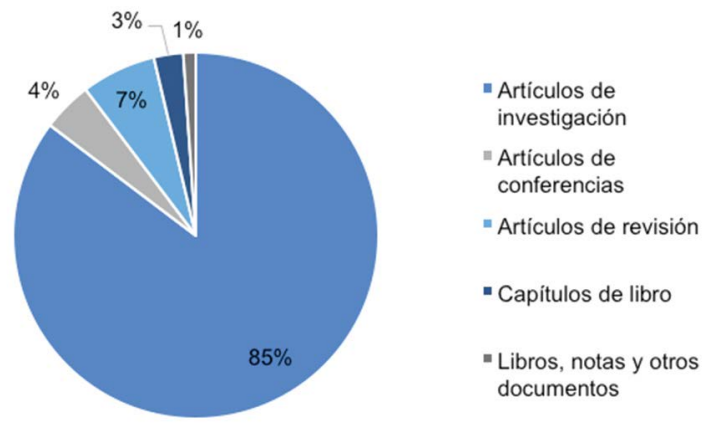

Fuente: Los autores

Por otra parte, una depuración de las publicaciones en la que se retiraron los estudios que no correspondían al objeto de estudio, arrojo un número de publicaciones relevantes de 22.907, distribuidas en diferentes áreas temáticas, Fig. 5. 
Fig. 5. DISTRIBUCIÓN PORCENTUAL DE DOCUMENTOS SEGÚN ÁREA DE ESTUDIO.

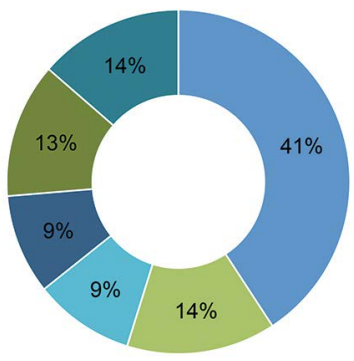

$$
\begin{aligned}
& \text { - Ciencias ambientales } \\
& \text { " Ciencias agrícolas y } \\
& \text { biológicas } \\
& \text { " Inmunología y microbiología } \\
& \text { - Bioquímica, genética y } \\
& \text { biología molecular } \\
& \text { - Química e ingeniería química } \\
& \text { - Otros }
\end{aligned}
$$

Fuente: Los autores

En el caso de Colombia, la investigación científica relacionada a la recuperación del recurso suelo se ha desarrollado desde 2006, con un número total de publicaciones igual a 68 , que se comparten con afiliaciones de España, Alemania, México, Estados Unidos, entre otros. Durante los últimos 5 años se ha aumentado el número de divulgaciones, representando el $58 \%$ del total de documentos relacionados. Estas investigaciones se desarrollan principalmente en: Universidad de Los Andes, Universidad de Córdoba, Universidad de Antioquia y Universidad Nacional de Colombia; con más de 5 documentos cada una. Por otra parte, la publicación con mayor número de citas se titula: "Compost supplementation with nutrients and microorganisms in composting process", investigación desarrollada por el centro de investigación y desarrollo conocido como Planta de bioprocesos y agroindustrial de la Universidad de Caldas.

\subsection{Evaluación patento métrica en ESPACENET}

El criterio de selección de la mejor ecuación de búsqueda

\begin{tabular}{|c|c|}
\hline Ecuación & Resultado \\
\hline $\begin{array}{l}\text { 1. ("soils" AND ("C05G3" AND "H01L21" AND "B09C1" } \\
\text { AND"C12N1" AND "A61K31")) }\end{array}$ & 179.368 \\
\hline 2. (“C05G3" AND “B09C1") & 380 \\
\hline 3. (“C12N1" AND “H01L21") & 34 \\
\hline 4. ("C05G3" AND “C12N1") & 1.508 \\
\hline
\end{tabular}
fue igual al empleado para la búsqueda de documentos en Scopus. Las ecuaciones de búsqueda utilizadas se muestran en la Tabla III. La seleccionada para el caso de las patentes, fue la número 2 , estas publicaciones resultan relevantes de acuerdo con el objeto del estudio.

TABLA III.

ECUACIONES DE BÚSQUEDA EVALUADAS EN ESPACENET.

Para el caso de las patentes, se observó que el país con mayor número de patente de datos en ESPACENET es China, seguido por Estados Unidos, Fig. 6, sin embargo, muchas de las patentes están construidas por más de un país, lo que modifica la representatividad de cada país.
Fig.6. PRODUCCIÓN DE PATENTES POR PAÍS.

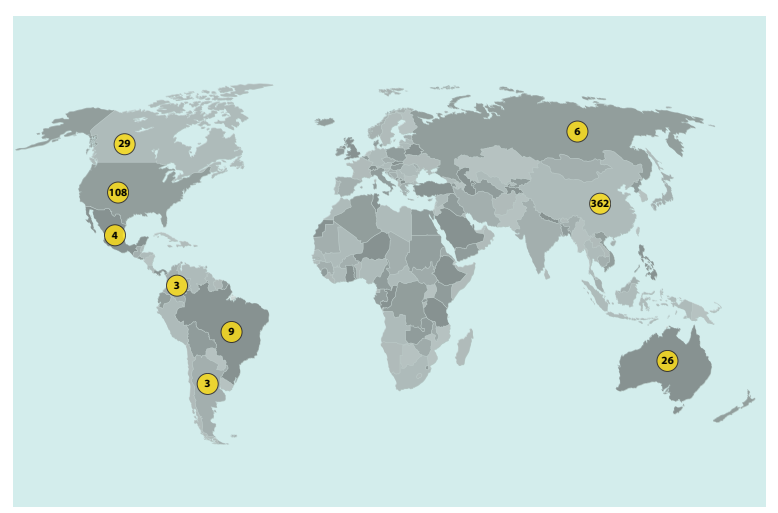

Fuente: Los autores

Asimismo, se observó que el registro de patentes se realiza desde 1970, con un auge desde 2010. Lo que coincide con el histograma de artículos académicos relacionados al tema de estudio, Fig.7.

Fig. 7. HISTÓRICO DE PUBLICACIÓN DE PATENTES.

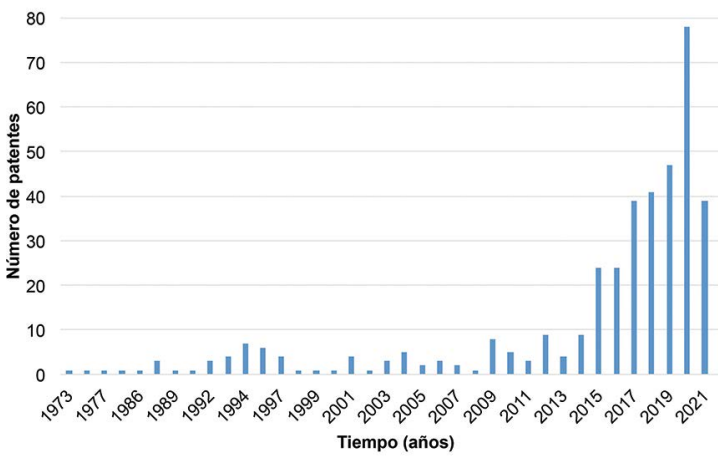

Fuente: Los autores

Se encontró un total de 100 inventores, que cuentan con un promedio de 2.24 patentes, afiliados a diferentes países. El inventor Miao Qingling es uno de los más relevantes, cuenta con 4 patentes relacionadas a la recuperación de suelos contaminados con metales pesados. El inventor Miao Quingling propone ciclos de biorremediación, que consisten en: uso de insecticidas, enriquecimiento y remediación por lombrices de tierra, y una descomposición microbiana. Se propone un proceso de dos meses en tres ciclos anuales de marzo a septiembre, estos ciclos garantizarían la remediación y reducirían los riegos de contaminación secundaria.

\subsection{Estudios desarrollados en relación con tecnologías para la recuperación de suelos}

Hay un gran número de investigaciones relacionadas a tecnologías para la recuperación de suelos. A continuación, se presentarán algunos de los estudios con mayor relevancia, esto de acuerdo con la ecuación de búsqueda seleccionada. 
Song U., 2020 estudio macrófitas que se emplean en la fitorremediación de rellenos sanitarios, Phragmites australis y Typha angustifolia, como substratos en procesos de compostaje. El objeto de la investigación era emplear esta vegetación, que venía de un proceso de bioacumulación de contaminantes, para aprovecharlas como potenciales fertilizantes o como material compostable. Encontraron que a pesar de que la tasa de descomposición de las macrófitas era pequeña, estas resultaban co-sustrato apropiado para proceso de compostaje ya implementados. Asimismo, el compost producido mejoro significativamente las condiciones del suelo estudiado, mejorando el crecimiento de plantas sin generar elevada acumulación de material toxico [13].

Reddy K.R. y Chirakkara R.A., 2019 estudiaron el efecto de una fitorremediación mejorada en un suelo de contaminación mixta ( $\mathrm{Pb}, \mathrm{Cr}$ y HAPs). Los suelos empleados se tomaron de ambientes históricamente contaminados, y las plantas empleadas fueron Avena sativa y Helianthus annuus. Encontraron que la remoción de Cr era mejor en Avena sativa, no hay movilización de $\mathrm{Pb}$ en ninguna de las plantas estudiadas, y la degradación de HAPs se presenta en periodos avanzados del crecimiento de las plantas. Los procesos de germinación, supervivencia y crecimiento de las plantas mejoraban al incluir enmiendas orgánicas y compost al suelo contaminado; empero esto no mejoro la degradación y/o remoción de contaminantes [14].

Chirakkara R.A. y Reddy K.R., 2015 realizaron un estudio que integraba el uso de enmiendas orgánicas (biochar, compost y soluciones de nutrientes) y enmiendas químicas (EDATA e Igepal CA-720) en procesos de fitorremediación con Helianthus annuus y Avena sativa en suelos con contaminación orgánica e inorgánica. Encontraron que los procesos de germinación y crecimiento en los suelos contaminados sin enmiendas eran más largos, además reducían la tasa de supervivencia de las plantas. El biochar y el compost mejoraron los procesos de crecimiento y acumulación de biomasa en las plantas, al igual que la remoción de $\mathrm{Cb}$ y $\mathrm{Pb}$; la remoción de $\mathrm{Cr}$ no se vio afectada por las enmiendas. Igualmente, mejoras en la degradación de HAPs se observaron con el uso de todas las enmiendas evaluadas [15].

Khan Z. y Anjaneyulu Y., 2006 estudiaron la aplicación del método de compostaje como una alternativa para la biorremediación de suelo y sedimento contaminado con fenoles, cloro/nitro fenoles y bencenos. Durante el proceso experimental se agregó compost comercial como inóculo, este permitió aumentar el número de microorganismos presentes en la pila a escala laboratorio. La máxima remoción de contaminantes se observó durante la fase de termofílica. El compost resultante podría emplearse como inóculo en procesos de descontaminación y/o como fertilizante [16].

\subsubsection{Colombia}

En el caso de Colombia, la última investigación publicada, que coincide con la ecuación de búsqueda, corresponde a una evaluación de la capacidad de fitorremediación de me- tales pesados $(\mathrm{Cd}+2$ y Hg+2) por Lolium perenne, incluyendo los efectos que puede causar los metales a la morfología, la producción de biomasa y la expresión genética de la planta. Encontraron que Lolium perenne cuenta con mecanismos que la hacen tolerante a la acumulación del contaminante. Se estableció una concentración máxima de 0.1 mg L-1 relacionada al crecimiento de las semillas, a esta concentración se evidencian cambios en la expresión genética relacionados a la respuesta al estrés causado por los metales [17].

Cabe resaltar la relevancia de esta investigación en el contexto colombiano, pues al ser un país agroindustrial, que busca cumplir con regulaciones que permitan la exportación de su producción agrícola; requiere generar alternativas que permitan el cultivo en suelos libres de tóxicos que puedan afectar la producción. Un ejemplo de ello es la acumulación de Cd en el cacao, que ha causado la modificación de regulaciones sobre los niveles máximos de Cd en los granos de cacao y productos derivados [18].

\section{CONCLUSIONES}

El análisis cienciométrico demuestra la relevancia que tiene la nación China en la generación de métodos de remediación de suelos, especialmente en procesos dirigidos a generar suelos de uso agrícola. Así mismo, se destaca la investigación desarrollada por Estados Unidos, quienes igualmente cuentan con un alto desarrollo agroindustrial. Las patentes de Colombia asociadas a la ecuación de búsqueda seleccionada no cuentan con relación directa a técnicas de remediación de suelos, pues se relaciona a procesos de control de plagas y fertilización agrícola. Sin embargo, para el caso de la investigación científica, se destaca el desarrollo de procesos de fitorremediación; donde las publicaciones se asocian a revistas Q1 con índice H superior a 180. En relación con lo anterior, es importante que se generen procesos colaborativos con grupos de investigación chinos y estadounidenses que permitan generar proceso de transferencia tecnológica asociada a la recuperación de suelos.

\section{REFERENCIAS}

[1] A. A. Juwarkar, S. K. Singh, and A. Mudhoo, "A comprehensive overview of elements in bioremediation," Reviews in Environmental Science and Biotechnology, vol. 9, no. 3, pp. 215-288, 2010, doi: 10.1007/s11157-010-9215-6.

[2] R. Lal et al., "Soils and sustainable development goals of the United Nations: An International Union of Soil Sciences perspective," Geoderma Regional, vol. 25, p. e00398, Jun. 2021, DOI: https://doi.org/ 10.1016/j.geodrs.2021.e00398.

[3] M. Deb and S. C. Sarkar, "Soil: An Essential but Somewhat Neglected Natural Resource," in Minerals and Allied Natural Resources and their Sustainable Development: Principles, Perspectives with Emphasis on the Indian Scenario, M. Deb and S. C. Sarkar, Eds. Singapore: Springer Singapore, 2017, pp. 421-442. DOI: https://doi.org/10.1007/978-981-10-4564-6_7

[4] D. C. Adriano, A. Chlopecka, and D. I. Kaplan, Role of soil chemistry in soil remediation and ecosystem conservation. 2015. DOI: https://doi.org/10.2136/sssaspecpub52.c13. 
[5] K. T. Osman, Soil degradation, conservation and remediation, vol. 9789400775909. 2014 DOI: https://doi.org/10.1007/978-94-007-7590-9.

[6] M. Ike, M. Yamashita, and S. Soda, Handbook of metal biotechnology: Applications for environmental conservation and sustainability. 2011. DOI: https://doi.org/10.4032/9789814267991.

[7] M. A. Ayub et al.,"Restoration of Degraded Soil for Sustainable Agriculture," in Soil Health Restoration and Management, R. S. Meena, Ed. Singapore: Springer Singapore, 2020, pp. 31-81. DOI: https://doi.org/10.1007/978-981-13-8570-4_2.

[8] Comité técnico CTN 166, "Gestión de la I+D+i: Sistema de vigilancia e inteligencia," Asociación Española de Normalización UNE 166006, Madrid, España, 2018.

[9] R, Hernández Sampieri; P, Baptista Lucio, Metodología de la investigación. México, D.F: McGraw-Hill, 2014.

[10] J. O. Alexander, "Library Applications," in Encyclopedia of Information Systems, H. Bidgoli, Ed. New York: Elsevier, 2003, pp. 55-76. DOI: https://doi.org/10.1016/B0-12-227240-4/00104-0.

[11] C. N. Mulligan, R. N. Yong, and B. F. Gibbs, "Remediation technologies for metal-contaminated soils and groundwater: An evaluation," Engineering Geology, vol. 60, no. 1-4, pp. 193-207, 2001, DOI: https://doi.org/10.1016/S0013-7952(00)00101-0.

[12] S. Ye et al., "Biological technologies for the remediation of co-contaminated soil," Critical Reviews in Biotechnology, vol. 37, no. 8, pp. 1062-1076, 2017, DOI: https://doi.org/10.1080/07388551.2017.1304357.
[13] U. Song, "Improvement of soil properties and plant responses by compost generated from biomass of phytoremediation plant," Environmental Engineering Research, vol. 25, no. 5, pp. 638-644, 2020, DOI: https://doi.org/10.4491/eer.2019.59.

[14] K. R. Reddy and R. A. Chirakkara, "Phytoremediation of field soil with mixed contamination," Environmental Science and Engineering, pp. 624-629, 2019, DOI: https://doi.org/10.1007/978-981-13-2221-1_68.

[15] R. A. Chirakkara and K. R. Reddy, "Biomass and chemical amendments for enhanced phytoremediation of mixed contaminated soils," Ecological Engineering, vol. 85, pp. 265274, 2015, DOI: https://doi.org/10.1016/j.ecoleng.2015.09.029.

[16] Z. Khan and Y. Anjaneyulu, "Bioremediation of contaminated soil and sediment by composting," Remediation, vol. 16, no. 4, pp. 109-122, 2006, doi: 10.1002/rem.20105.

[17] Y. Cruz et al., "Gene expression and morphological responses of Lolium perenne L. exposed to cadmium $(\mathrm{Cd} 2+)$ and mercury (Hg2+)," Scientific Reports, vol. 11, no. 1, 2021, DOI: https://doi.org/10.1038/s41598-021-90826-y.

[18] A. P. Ferreira de Oliveira, R. F. Milani, P. Efraim, M. A. Morgano, and S. A. V. Tfouni, "C $\mathrm{Cd}$ and $\mathrm{Pb}$ in cocoa beans: Occurrence and effects of chocolate processing," Food Control, vol. 119, 2021, DOI: https://doi.org/10.1016/j.foodcont.2020.107455. 